\title{
Dynamic Range Improvement of New Leap-Frog Filter Using Numerical Optimization
}

\author{
Drazen Jurisic and Neven Mijat \\ Faculty of Electrical Engineering and Computing \\ University of Zagreb \\ Unska 3, HR-10000 Zagreb, Croatia \\ E-mail: \{drazen.jurisic, neven.mijat\}@fer.hr
}

\author{
George S. Moschytz \\ Faculty of Engineering \\ Bar-Ilan University \\ IL-52900 Ramat-Gan, Israel \\ E-mail: George-S.Moschytz@biu.ac.il
}

\begin{abstract}
In this paper the dynamic-range optimization of a new class of low-pass active- $R C$ filters, based on the leap-frog topology is presented, using the MATLAB Optimization Toolbox. The new structure is a simplified leap-frog structure with a reduced number of components, reduced complexity, and permitting a straightforward design procedure. It is most suitable for the nonbalanced-to-ground version of low-sensitivity filters. As an illustration of the efficiency of the proposed new leap-frog filter, a sensitivity analysis using Monte Carlo runs was performed on examples with Butterworth and Chebyshev $0.5 \mathrm{~dB}$ pass-band ripple low-pass filters. A comparison with other common filters such as standard leap-frog filters and single-amplifier Biquads demonstrates the sensitivity improvement obtained with the new filters. In this paper we present a numerical method of optimizing these new filters for improved dynamic range.
\end{abstract}

\section{INTRODUCTION}

Since the most critical disadvantage of active- $R C$ filters is their high sensitivity to component tolerances, new proposals of lowsensitivity circuits are generally welcome. A new topology for an active-RC leap-frog filter was published recently [1][2]. Compared to standard leap-frog filters it has a lower complexity and a lower number of components, while keeping a low sensitivity to component tolerances (see also [3]).

In this paper we present a method of optimizing the dynamic range of the new leap-frog filters (NLF). Unlike the case with standard leap-frog filters (LF), which can use signal-flow-graph methods (SFG) for dynamic range optimization [4] it can be shown that in the case of the NLF this is not possible. In this paper, we show that a dynamic range optimization of the NLF can be achieved using a numerical optimization in conjunction with MATLAB [5]. The optimization method is illustrated with a third- and fourth-order Butterworth and Chebyshev $0.5 \mathrm{~dB}$ filter. The sensitivity of the dynamic-range-optimized NLF filters is compared with two common filter circuits, a standard leap frog (LF) and a single-amplifier Biquad (SAB) by Monte-Carlo analysis, using the Cadence PSpice 16 program [6].

\section{NEW LEAP-Frog FILTERS}

Consider the NLF low-pass (LP) filters with a reduced number of amplifiers (in the nonbalanced-to-ground mode) shown in Figs. 1-4. The reduced number of amplifiers refers to the fact that, compared to the standard LF filter, the NLF equivalent requires no inverter amplifiers. (In a fully balanced-to-ground realization this advantage disappears because there is no need for an inverter since signals from negative outputs of differential-input-differential-output opamps are generally available.)

\section{A. Third-Order Filters}

As will be demonstrated below, in order to adjust the voltages $V_{1}$ to $V_{3}$ for maximum dynamic range at the amplifier outputs, additional resistive dividers in the feedback loops of the filter in Fig. 1 (originally introduced in [1]) are included as shown in Fig. 2. The attenuation factors thereby obtained are denoted by $\beta_{1}=R_{0} /\left(R_{0}+R_{01}\right)$, $\beta_{2}=R_{0} /\left(R_{0}+R_{02}\right)$, and the ratio $\alpha=R_{3} / R_{4}$. In Section $3, \beta_{1}, \beta_{2}$ and $\alpha$ will be used for the optimization process.

The voltage transfer function of a third-order allpole LP filter, in terms of the polynomial coefficients $a_{i}(i=0,1,2)$, is given by

$$
H(s)=\frac{V_{\text {out }}(s)}{V_{\text {in }}(s)}=\frac{k \cdot a_{0}}{s^{3}+a_{2} s^{2}+a_{1} s+a_{0}},
$$

where, in the case of the filter in Fig. 2, the coefficients are given by

$$
\begin{aligned}
& a_{0}=\alpha \beta_{1} \omega_{1} \omega_{2} \omega_{3} ; \\
& a_{1}=\left(\alpha \beta_{1}+\beta_{2}\right) \omega_{2} \omega_{3}+\beta_{1} \omega_{1} \omega_{2} ; \\
& a_{2}=\left(\alpha+\beta_{2}\right) \omega_{3}+\beta_{1} \omega_{2} ; \\
& k=-1 /\left(\alpha \beta_{1}\right),
\end{aligned}
$$

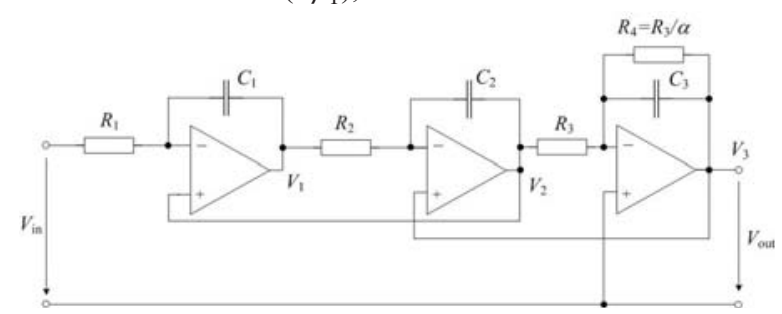

Figure 1. New third-order LP leap-frog filter (NLF)

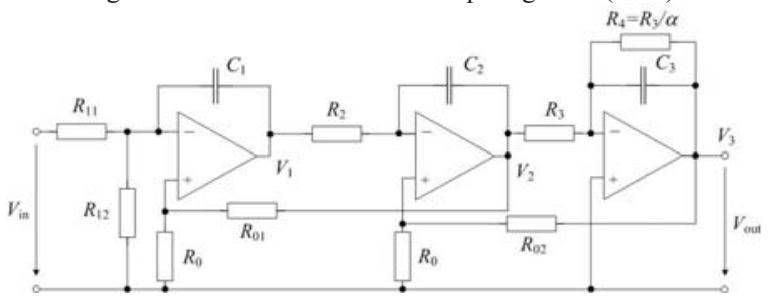

Figure 2. Third-order LP filter for dynamic range optimization (NLFO).

This work was supported by the Ministry of Science, Education and Sports of Republic of Croatia. 
and the parameters $\omega_{i}$ are reciprocals of the $R C$-products, defined by:

$$
\omega_{i}=\left(R_{i} C_{i}\right)^{-1}(i=1,2,3) .
$$

Obviously, if $\alpha=0$, the resistance $R_{4}$ becomes infinite. Thus, from (2), $a_{0}=0$, which means that the real pole of the transfer function is actually a pole at the origin. Consequently, for an odd-order filter, in order to realize a negative real pole, $\alpha$ must always be non-zero and $R_{4}<\infty$. The parameters $\omega_{i}(i=1,2,3)$ can be obtained by solving the following equations [which follow from (2)]:

$$
\begin{aligned}
& \omega_{3}^{3}-\omega_{3}^{2} \frac{a_{2}}{\alpha+\beta_{2}}+\omega_{3} \frac{a_{1}}{X}-\frac{a_{0}}{\alpha X}=0, \\
& \text { where } X=\left(\alpha+\beta_{2} / \beta_{1}\right)\left(\alpha+\beta_{2}\right) ; \\
& \omega_{2}=\frac{a_{2}-\left(\alpha+\beta_{2}\right) \omega_{3}}{\beta_{1}} ; \omega_{1}=\frac{a_{0}}{\beta_{1} \alpha \omega_{2} \omega_{3}} .
\end{aligned}
$$

Finally, selecting suitable values (depending on the technology used) for $C_{1}, C_{2}$ and $C_{3}$, and with the obtained values of $\omega_{i}$, the resistors $R_{i}(i=1,2,3)$ readily follow from (3):

$$
R_{i}=\left(\omega_{i} C_{i}\right)^{-1}(i=1,2,3) .
$$

Note that, when $\beta_{1}=\beta_{2}=1$, the filter circuit in Fig. 2 simplifies to that of Fig. 1, and the design equations (4) have a simpler form and can be used to design the filter in Fig. 1.

\section{B. Fourth-Order Filters}

As an example of an even-order NLF filter, consider the two fourth-order filters shown in Figs. 3 and 4. Note that the (simpler) filter in Fig. 3 does not need $R_{5}$ (i.e. $\alpha=0$ ) because the coefficient $a_{0}$ [see (7) below] does not contain the factor $\alpha$. This holds for all evenorder NLF filters. As in the previous (third-order) case, the filter in Fig. 4, which is a modified version of the filter in Fig. 3, requires additional feedback resistors and an additional resistor $R_{5}$ for dynamic range optimization. This is because, in the optimization process for the fourth-order filter, we need four free design parameters, i.e. $\beta_{1}, \beta_{2}, \beta_{3}$ and $\alpha$.

The fourth-order LP voltage transfer function, in terms of the polynomial coefficients $a_{i}(i=0,1,2,3)$, is given by

$$
H(s)=\frac{V_{\text {out }}(s)}{V_{\text {in }}(s)}=\frac{k \cdot a_{0}}{s^{4}+a_{3} s^{3}+a_{2} s^{2}+a_{1} s+a_{0}},
$$

where, in the case of the filter in Fig. 4, the coefficients are given by

$$
\begin{aligned}
& a_{0}=\beta_{1} \beta_{3} \omega_{1} \omega_{2} \omega_{3} \omega_{4} ; \\
& a_{1}=\left[\beta_{1}\left(\alpha+\beta_{3}\right) \omega_{1}+\left(\alpha \beta_{2}+\beta_{1} \beta_{3}\right) \omega_{3}\right] \omega_{2} \omega_{4} ; \\
& a_{2}=\left(\beta_{1} \omega_{1}+\beta_{2} \omega_{3}\right) \omega_{2}+\left[\beta_{1}\left(\alpha+\beta_{3}\right) \omega_{2}+\left(\alpha \beta_{2}+\beta_{3}\right) \omega_{3}\right] \omega_{4} ; \\
& a_{3}=\beta_{1} \omega_{2}+\beta_{2} \omega_{3}+\left(\alpha+\beta_{3}\right) \omega_{4} ; \\
& k=1 /\left(\beta_{1} \beta_{3}\right) .
\end{aligned}
$$

It can be shown that the parameters $\omega_{i}(i=1,2,3,4)$ can be calculated only by solving the equations in (7) numerically, e.g. by using MATLAB. To start, we select values for $\beta_{1}, \beta_{2}, \beta_{3}, \alpha$ and then calculate parameters $\omega_{i}(i=1,2,3,4)$. We do this by equating the expressions on the right hand side in (7) with the coefficients $a_{i}(i=1$, $2,3,4)$ which are given in Table I.

As an illustration of the design process for dynamic range optimization, consider the following two examples of a third- and fourth-order LP filter transfer function with the cut-off frequency of 1kHz: (i) Butterworth and (ii) Chebyshev with the pass-band ripple $A_{\max }=0.5 \mathrm{~dB}$. The coefficients $a_{i}(i=1,2,3,4)$ are given in Table I. Note that a Butterworth filter, which corresponds to the limit case of a zero-ripple Chebyshev filter of equal order, always has lower pole Qs than the latter.

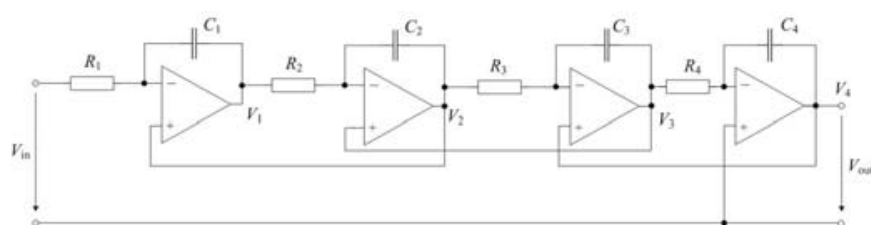

Figure 3. New fourth-order LP leap-frog filter (NLF).

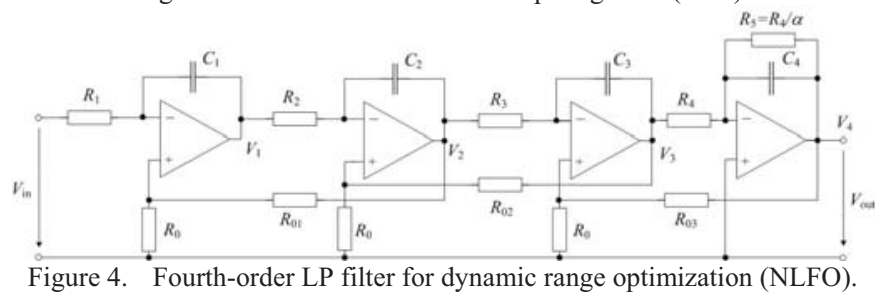

TABLE I. THIRD- AND FOURTH-ORDER BUTT. AND CHEBY.

TRANSFER-FUNCTION COEFFICIENTS WITH $1 \mathrm{KHZ}$ CUT-OFF FREQUENCY.

\begin{tabular}{|l|l|l|l|l|}
\hline \multirow{2}{*}{ COEFFICIENT } & \multicolumn{2}{|c|}{ Butterworth } & \multicolumn{2}{c|}{ Chebyshev 0.5dB } \\
\cline { 2 - 5 } & Third-order & Fourth-order & Third-order & Fourth-order \\
\hline$a_{0}$ & $2.4805 \cdot 10^{11}$ & $1.55855 \cdot 10^{15}$ & $1.7753 \cdot 10^{11}$ & $5.90768 \cdot 10^{15}$ \\
\hline$a_{1}$ & $7.8957 \cdot 10^{7}$ & $6.48186 \cdot 10^{11}$ & $6.0595 \cdot 10^{7}$ & $2.54364 \cdot 10^{11}$ \\
\hline$a_{2}$ & $1.2566 \cdot 10^{4}$ & $1.34788 \cdot 10^{8}$ & $7.8723 \cdot 10^{3}$ & $6.77792 \cdot 10^{7}$ \\
\hline$a_{3}$ & 16418.8 & & 7523.4 \\
\hline
\end{tabular}

III. DYNAMIC-RANGE OPTIMIZATION

\section{A. Third-Order NLF Filter Optimization}

For the filter in Fig. 2, the voltage transfer function from the filter input to every opamp output is defined by:

$$
H_{i}(s)=\frac{V_{i}(s)}{V_{\text {in }}(s)}=\frac{N_{i}(s)}{D(s)} ; \quad(i=1,2,3),
$$

where:

$$
\begin{aligned}
& N_{1}(s)=-\omega_{1}\left[s^{2}+\left(\beta_{2}+\alpha\right) \omega_{3} s+\omega_{2} \omega_{3} \beta_{2}\right] \\
& N_{2}(s)=\left(s+\alpha \omega_{3}\right) \omega_{1} \omega_{2} ; \\
& N_{3}(s)=-\omega_{1} \omega_{2} \omega_{3} .
\end{aligned}
$$

Note that all the transfer functions in (8) have the same denominator $D(s)=s^{3}+a_{2} s^{2}+a_{1} s+a_{0}$, with coefficients $a_{i}(i=1,2,3)$ from Table I.

The amplitude-frequency characteristics of the transfer functions in (8), i.e. $A_{i}(\omega)[\mathrm{dB}]=20 \log \left|H_{i}(j \omega)\right|(i=1,2,3)$, were simulated using Cadence Pspice 16 for the circuit in Fig. 1 , and $\alpha=\beta_{1}=\beta_{2}=1$. In all simulations we use a voltage-controlled voltage source (VCVS) E with a high gain of $10^{6}$ to represent an ideal opamp. They are shown in Figs. 5(a)-(b) for the Butterworth and Chebyshev examples, respectively.

The design values and components of the non-optimized filter in Fig. 1 readily follow from (4); they are the same as the examples in [1], denormalized to $1 \mathrm{kHz}$. As can be seen in Fig. 5(a) and (b) the magnitude of inner opamp outputs have maximum values other than $0 \mathrm{~dB}$ (all maxima are shown). The maximum of $A_{3}(\omega)$ is at $0 \mathrm{~dB}$.

One often-used criterion that is useful to guarantee maximum dynamic range is: for a given input signal equal to $1 \mathrm{~V}$, the largest signal anywhere within the circuit (i.e. at every opamp output) should be equal to $1 \mathrm{~V}$. In other words, the maximum gain anywhere within the circuit should be equal to $O d B$ (see [4] Section 6).

As pointed out earlier, simple signal-level scaling by scaling the corresponding nodes in the equivalent SFG (e.g. [7]) is not possible for the NLF filter. One reason for this is that using the SFG method, the factor by which each node is to be scaled must be known in advance. In our case the scaling factor is calculated only at the end of the optimization process. 

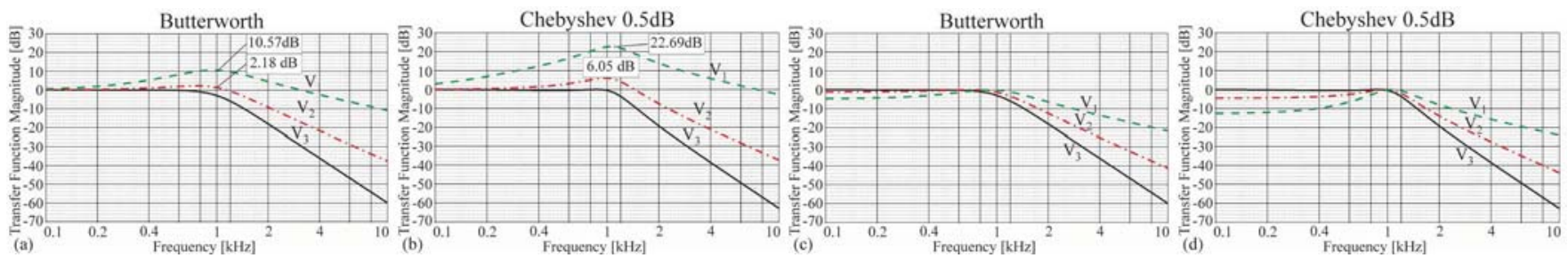

Figure 5. PSpice magnitude response of the new leap-frog third-order LP filters at the opamps' outputs. (a)-(b): non-optimized. (c)-(d): optimized.

Thus, it turns out that the only way to optimize the dynamic range of the NLF filter is to determine the free design parameters $\alpha$, $\beta_{1}$, and $\beta_{2}$ for equal maxima of $A_{i}(\omega)[\mathrm{dB}]$; and this can be accomplished only by using a method of numerical optimization. In doing so, we define a quantity $\varepsilon$, which represents the sum of the squares of the distances between the magnitude maxima and the arithmetical mean of those maxima defined by $z$, which is (in our three-amplifier case) given by:

$$
z=\frac{1}{3} \sum_{i=1}^{3}\left|H_{i}(j \omega)\right|_{\max } .
$$

Thus, the error of the desired equal-maxima will be given by:

$$
\varepsilon=\sum_{i=1}^{3}\left(\left|H_{i}(j \omega)\right|_{\max }-z\right)^{2} .
$$

The error $\varepsilon$ is used as a 'goal' (or 'cost') function in the optimization process. The optimum design parameters $\alpha, \beta_{1}$, and $\beta_{2}$ are determined by searching the minimum of the equal- maxima error in (11), i.e.

$$
\mathbf{x}_{o p t}=\underset{\mathbf{x}}{\arg \min } \varepsilon,
$$

where:

$$
\mathbf{x}=\left[\begin{array}{lll}
\alpha & \beta_{1} & \beta_{2}
\end{array}\right]^{T} .
$$

To find $\left|H_{i}(j \omega)\right|_{\max }(i=1,2,3)$ in $(10)$ and (11), we first have to find the frequencies at which the signal maxima occur. This can be done using first derivatives, i.e.

$$
\frac{d\left|H_{i}(j \omega)\right|^{2}}{d \omega}=0(i=1,2,3) .
$$

Derivations can be calculated inside the main MATLAB .m file written for optimization, using the 'Symbolic Math Toolbox' in MATLAB [8]. To search for the minimum in (12) numerically, a 'Quasi-Newton line search' method was used, performing the unconstrained minimization of the goal function (11) [5]. For this task we used the function fminunc of the 'Otimization Toolbox' in MATLAB [9]. Starting from the vector $\mathbf{x}_{\text {start }}=\left[\begin{array}{lll}0.9 & 0.3 & 0.6\end{array}\right]^{T}$, we obtain the solution

$$
\mathbf{x}_{\text {opt }}=\left[\begin{array}{lll}
0.87223441 & 0.28818426 & 0.57509193
\end{array}\right]^{T},
$$

and starting from $\mathbf{x}_{\text {start }}=\left[\begin{array}{lll}0.5 & 0.3 & 0.3\end{array}\right]^{T}$ we obtain

$$
\mathbf{x}_{\text {opt }}=\left[\begin{array}{lll}
0.59086092 & 0.31040132 & 0.23091202
\end{array}\right]^{T} \text {, }
$$

for the Butterworth and Chebyshev filter, respectively. After 12 iterations the precision of $\varepsilon \approx 10^{-13}$ was achieved, which is more than sufficient for our optimization problem.

All three maxima converged to $z$, which represents the d.c. gain of the final filter circuit: $A_{0 B u t}=11.9939 \mathrm{~dB}$ for the Butterworth and $A_{0 \text { Cheby }}=14.7318 \mathrm{~dB}$ for the Chebyshev filter, respectively. To realize the maximum $0 \mathrm{~dB}$ of the overall transfer function magnitude, the input resistor $R_{1}$ is split into two resistors $R_{11}$ and $R_{12}$ (see Fig. 2). The design values of the optimized filters are $\omega_{1}=20221, \omega_{2}=6628.1$, $\omega_{3}=7362.7(\mathrm{rad} / \mathrm{s})$ for the Butterworth, and $\omega_{1}=21301, \omega_{2}=6317.2$, $\omega_{3}=7193.5(\mathrm{rad} / \mathrm{s})$ for the Chebyshev filter, respectively. A simple check for the accuracy of the calculated results is to include all obtained values ( $\omega \mathrm{s}, \alpha \mathrm{s}$ and $\beta \mathrm{s})$ in (2), and to compare the results with the coefficients that we started out with in Table I. The corresponding optimized magnitudes of the three opamp outputs are shown in Fig. 5 (c)-(d).

Depending on the selection of the starting point $\mathbf{x}_{\text {start }}$ the procedure will lead to an acceptable global minimum, i.e. for which all $\alpha$ s and $\beta$ s are positive, and the $\beta$ s are less than or equal to unity. Choosing another starting vector $\mathbf{x}_{\text {start }}$ will either give different values of maxima, or no global minimum, at all.

\section{B. Fourth-Order NLF Filter Optimization}

As with the third-order NLF filter, here we proceed with the design of the optimum fourth-order NLF filter shown in Fig. 4. Again, we have to apply numerical calculations of the filter components, symbolic calculations to find the maxima of the amplitude response, and a numerical optimization to find the optimum parameters $\beta_{1}, \beta_{2}, \beta_{3}$ and $\alpha$. Starting from vector $\mathbf{x}_{\text {start }}=$ $\left[\begin{array}{llll}1 & 0.3 & 0.3 & 0.8\end{array}\right]^{T}$ we obtain the solution

$\mathbf{x}_{\text {opt }}=\left[\begin{array}{lllll}0.85001732 & 0.40126466 & 0.28161741 & 0.69535836\end{array}\right]^{T}$, and from $\mathbf{x}_{\text {start }}=\left[\begin{array}{llll}0.4 & 0.2 & 0.2 & 0.4\end{array}\right]^{T}$ we obtain

$\mathbf{x}_{\text {opt }}=\left[\begin{array}{llll}0.46553270 & 0.21256312 & 0.10217718 & 0.36567503\end{array}\right]^{T}$, for the Butterworth and Chebyshev filter, respectively.

The corresponding parameters are $\omega_{1}=15911.5, \omega_{2}=6159.32$, $\omega_{3}=7429.69, \omega_{4}=7671.21(\mathrm{rad} / \mathrm{s})$ and $A_{0 B u t t}=11.0872 \mathrm{~dB}$ for the Butterworth, and $\omega_{1}=30299.8, \omega_{2}=6189.65, \omega_{3}=6023.54, \omega_{4}=$ $6727.84(\mathrm{rad} / \mathrm{s})$, and $A_{0 \text { Cheby }}=22.6883 \mathrm{~dB}$ for the Chebyshev filter, respectively. The magnitude at the amplifier outputs of the optimized filter are shown in Fig. 6(c)-(d). The non-optimized filter, with the magnitudes in Fig. 6(a)-(b), were calculated starting from $\alpha=0$ and $\beta_{1}=\beta_{2}=\beta_{3}=1$. They were realized using the filter circuit in Fig. 3 .

The proposed optimization method is universal in that it can be applied to other filter structures, e.g. filter cascade, follow-the-leader feedback, etc., as well as to other filter types, e.g. high- and band-pass filters. In doing so, the same steps as presented here have to be applied: (i) find maximum of amplitude response at the amplifier outputs; (ii) decide which elements have to be tuned to adjust those maxima; (iii) perform numerical optimization until all maxima are equal.

\section{SENSITIVITY ANALYSIS}

In what follows we compare the sensitivity of our fourth-order NLF filter to the sensitivity of two conventional filter circuits: (i) the standard leap frog (LF) in Fig. 7 and (ii) the single-amplifier Biquad (SAB) in Fig. 8. These were designed using the standard methods presented in [4] and [10], respectively. The values for the LF filter components follow from the filter tables in [11]. The SAB filter was designed using the impedance tapering method (see [12]) and therefore already has reduced sensitivity. All the filters are denormalized to the cut-off frequency of $1 \mathrm{kHz}$.

Using PSpice with Monte Carlo runs [6] (with zero-mean Gaussian distribution and $1 \%$ standard deviation of all passive components), the responses shown in Fig. 9 were obtained. For the comparison, all ordinate scales were selected to be the same. The new leap-frog filter shows very low sensitivity, as does the standard LF filter. The SAB filter has a larger sensitivity than either of the leapfrog filters. 

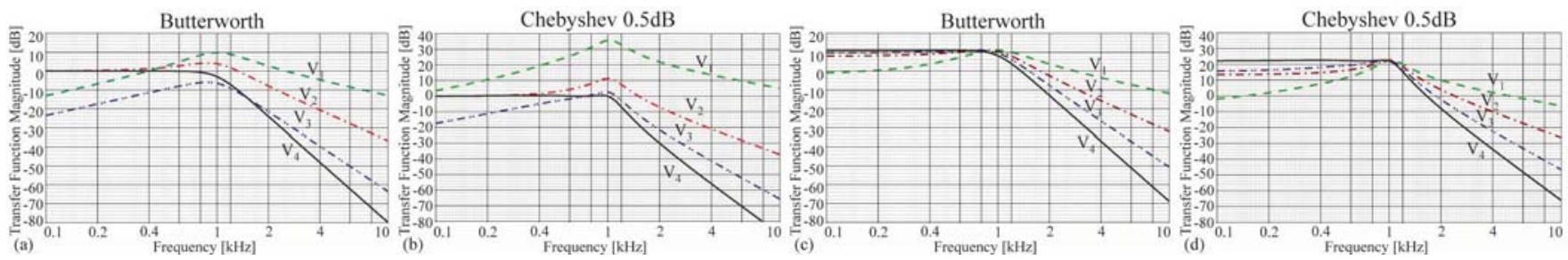

Figure 6. PSpice magnitude response of the new leap-frog fourth-order LP filter at the opamps' outputs. (a)-(b): non-optimized. (c)-(d): optimized.

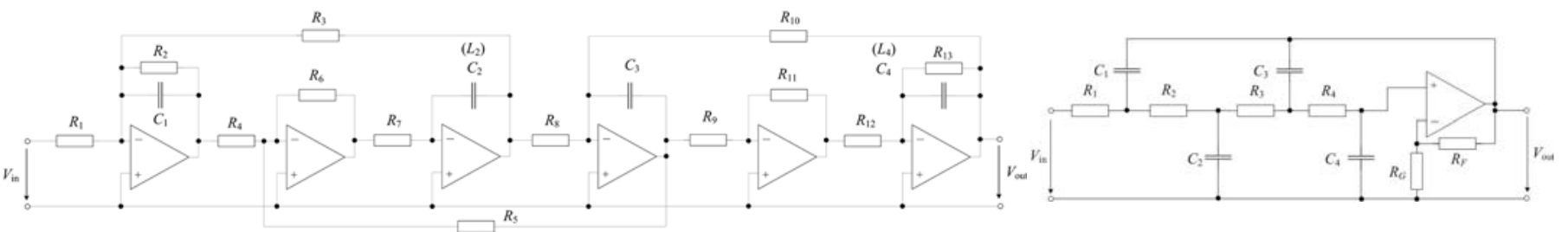

Figure 7. Fourth-order standard leap frog filter (LF).

Figure 8. Fourth-order SAB filter.
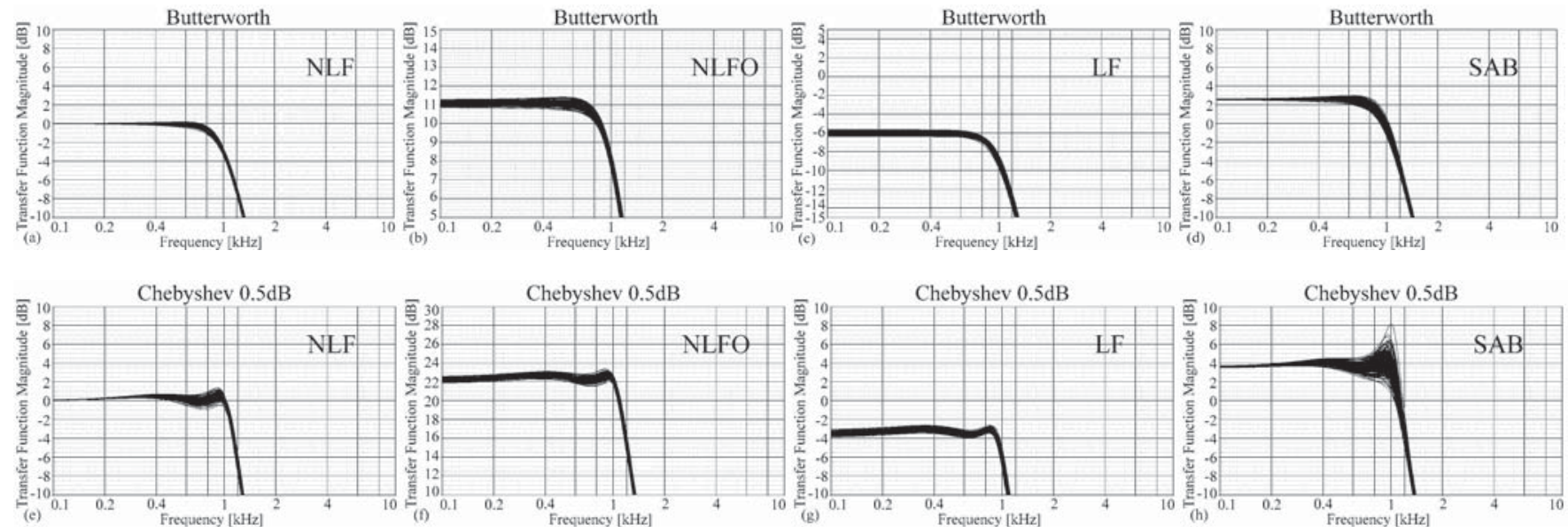

Figure 9. Monte Carlo runs of fourth-order filter examples: new leap frog (NLF); new leap frog optimized (NLFO); leap frog (LF); single-amplifier Biquad (SAB). (a)-(d): Butterworth; (e)-(h): Chebyshev 0.5dB

The sensitivity of the third-order NLF, LF and SAB circuits are compared in [1], and lead to the same conclusions.

\section{CONCLUSIONS}

This paper presents the dynamic-range optimization of the new leap-frog (NLF) topology used for the design of the allpole LP filters recently published in [1][2]. We demonstrate that the new leap-frog filter can be efficiently optimized for maximum dynamic range by using numerical optimization methods. More conventional methods, such as signal-flow-graph node scaling, are not possible for these filters. Optimizing the dynamic range of a filter is an important factor in improving the performance of a filter. The new filter has a canonical number of components. The presented topology is also shown to have low sensitivity to component tolerances-in some cases even lower than that of the standard leap-frog circuit. This is verified by MC sensitivity analysis. The new leap-frog topology can be generalized to realize $n^{\text {th }}$-order filters - as is the case for the standard leap-frog filter. Higher-order filters can be optimized for maximum dynamic range using the same methods as presented in this paper for the case of third- and fourth-order filters.

\section{REFERENCES}

[1] N. Mijat, D. Jurisic, and M. Ranilovic, "Novel low-sensitivity, thirdorder LP active leap-frog filter," Proceedings of the $33^{\text {rd }}$ MIPRO 2010 , Opatija, Croatia, pp. 161-164, May 2010.
[2] N. Mijat, D. Jurisic, and G. S. Moschytz, "A novel third-order leap-frog active filter," Automatika, in press. Available from: http://hrcak.srce.hr/automatika.

[3] H. J. Orchard, "Inductorless filters," Electronic Letters, vol. 2, no. 6, pp. 224-225, June 1966.

[4] R. Schaumann, M. S. Ghausi, and K. R. Laker, Design of Analog Filters, Passive, Active RC, and Switched Capacitor, Prentice Hall, New Jersey 1990.

[5] R. Fletcher, Practical Methods of Optimization, vol. 1, John Wiley and Sons, 1980.

[6] PSpice Users Guide ver. 16.5, Cadence Design Systems, Inc. (Cadence), San Jose CA-USA, May 2011.

[7] D. J. Perry, "Scaling transformation of multiple-feedback filters," Proceedings IEE, vol.128, pp.176-179, August 1981.

[8] MATLAB: Symbolic Math Toolbox, MathWorks, Inc., Natick, MA, 2010.

[9] MATLAB: Optimization Toolbox, MathWorks, Inc., Natick, MA, 2010.

[10] D. Jurisic, G. S. Moschytz, and N. Mijat, "Low-sensitivity, singleamplifier, active-RC allpole filters using tables," Automatika, vol. 49, no. 3-4, pp. 159-173, November 2008. Available from: http://hrcak.srce.hr/automatika.

[11] A. I. Zverev, Handbook of Filter Synthesis, John Wiley and Sons, New York 1967.

[12] G. S. Moschytz, "Low-sensitivity, low-power, active-RC allpole filters using impedance tapering," IEEE Transactions on Circuits and Systems-Part II, vol. CAS-46, no. 8, pp. 1009-1026, August 1999. 